\title{
Utility of Dynamic Susceptibility Contrast Perfusion-Weighted MR Imaging and "C-Methionine PET/CT for Differentiation of Tumor Recurrence from Radiation Injury in Patients with High-Grade Gliomas
}

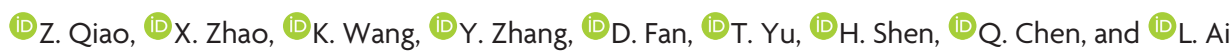

\begin{abstract}
BACKGROUND AND PURPOSE: Both ${ }^{11} \mathrm{C}$-methionine PET/CT and DSC-PWI could be used to differentiate radiation injury from recurrent brain tumors. Our aim was to assess the performance of MET PET/CT and DSC-PWI for differentiation of recurrence and radiation injury in patients with high-grade gliomas and to quantitatively analyze the diagnostic values of PET and PWI parameters.
\end{abstract}

MATERIALS AND METHODS: Forty-two patients with high-grade gliomas were enrolled in this study. The final diagnosis was determined by histopathologic analysis or clinical follow-up. PWI and PET parameters were recorded and compared between patients with recurrence and those with radiation injury using Student $t$ tests. Receiver operating characteristic and logistic regression analyses were used to determine the diagnostic performance of each parameter.

RESULTS: The final diagnosis was recurrence in 33 patients and radiation injury in 9. PET/CT showed a patient-based sensitivity and specificity of 0.909 and 0.556 , respectively, while PWI showed values of 0.667 and 0.778 , respectively. The maximum standardized uptake value, mean standardized uptake value, tumor-to-background maximum standardized uptake value, and mean relative CBV were significantly higher for patients with recurrence than for patients with radiation injury. All these parameters showed a high discriminative power in receiver operating characteristic analysis. The optimal cutoff values for the tumor-to-background maximum standardized uptake value and mean relative CBV were 1.85 and 1.83, respectively, and corresponding sensitivities and specificities for the diagnosis of recurrence were 0.97 and 0.667 and 0.788 and 0.88 , respectively. Areas under the curve for the tumor-to-background maximum standardized uptake value and mean relative CBV were $0.847 \pm 0.077$ and $0.845 \pm 0.078$, respectively. Combined assessment of the tumor-to-background maximum standardized uptake value and mean relative CBV showed the largest area under the curve $(0.953 \pm 0.031)$, with corresponding sensitivity and specificity of 0.848 and 1.0 , respectively.

CONCLUSIONS: Both ${ }^{11} \mathrm{C}$-methionine $\mathrm{PET} / \mathrm{CT}$ and $\mathrm{PWI}$ are equally accurate in the differentiation of recurrence from radiation injury in patients with high-grade gliomas, and a combination of the 2 modalities could result in increased diagnostic accuracy.

ABBREVIATIONS: $\mathrm{AUC}=$ area under the curve; $\mathrm{MET}={ }^{\mathrm{C}} \mathrm{C}$-methionine; $\mathrm{HGG}=$ high-grade glioma; max $=$ maximum; $\mathrm{rCBV}=$ relative $\mathrm{CBV}$; SUV = standardized uptake value; $\mathrm{TBR}=$ tumor-to-background

G liomas are the most common primary brain tumors. Tumor resection followed by postoperative chemotherapy and radiation therapy is the primary treatment for gliomas. However, radiation therapy may damage normal brain tissue and result in

Received May 29, 2018; accepted after revision November 24.

From the Departments of Nuclear Medicine (Z.Q., X.Z., K.W., Y.Z., D.F., Q.C., L.A.) and Radiology (H.S.), Beijing Tian Tan Hospital, Capital Medical University, Beijing China; Department of Medical Imaging (T.Y.), Cancer Hospital of China Medical University, Shenyang, China; and Department of Medical Imaging (T.Y.), Liaoning Cancer Hospital and Institute, Shenyang, China.

Zhen Qiao and Xiaobin Zhao contributed equally to this work and should be considered co-first authors

This work was supported by the Beijing Natural Science Foundation(7184207); the Clinical Capability Construction Project for Liaoning Provincial Hospitals (LNCCCB06-2014); the National Basic Research Program of China (2015CB755500); and the Youth Research Fund of Beijing Tian Tan Hospital (2016-YQN-02). adverse effects involving the brain. Classically, radiation injury can be classified into acute and delayed reactions. Radiation-induced necrosis is the most severe form of radiation injury and usually occurs 3-12 months after radiation therapy, though it can also occur years after treatment. The incidence of radiation-induced necrosis is reportedly $3 \%-24 \% .{ }^{1}$ The recurrence rate for gliomas, particularly high-grade gliomas (HGGs), is extremely high. The differentiation of tumor recurrence from radiation injury is an important part of clinical management. However, both recurrent active tumors and necrotic inflammation result in

Please address correspondence to Lin Ai, Beijing Tian Tan Hospital, Capital Medical University, 6, Tiantanxili, Beijing, 100050, China; e-mail: ailin@bjtth.org

-- Indicates open access to non-subscribers at www.ajnr.org

三 Indicates article with supplemental on-line table.

http://dx.doi.org/10.3174/ajnr.A5952 
breakdown of the BBB; therefore, both conditions show similar enhancement in gadolinium-enhanced MR imaging. As a result, discrimination between them can be challenging. More advanced imaging strategies have thus been developed, mainly advanced MR imaging techniques and novel PET radiotracers to target the biologic activity of tumor cells. ${ }^{2,3}$

PWI can demonstrate the characteristics of the vascular physiology and hemodynamics of tumors and has been used to differentiate recurrence from radiation injury. ${ }^{4-7}$ Dynamic contrast-enhanced MR imaging can quantitatively measure the permeability of immature microvessels in tumors by generating quantitative parameters such as the volumetric transfer constant, fractional plasma volume, and fractional volume of the extracellular extravascular space. Dynamic contrast-enhanced imaging is used less frequently in clinical practice. DSC-MR imaging is the most commonly used perfusion MR imaging technique in clinical practice; it is a standard method for measuring cerebral blood volume and cerebral blood flow. Because of treatment-induced vascular endothelial damage and coagulation necrosis, radiation injury is associated with hypoperfusion. Accordingly, the relative CBV (rCBV) tends to be lower with radiation necrosis than with tumor recurrence.

Amino acid PET is useful for the noninvasive differentiation of tumor and nontumoral lesions because tumors have significantly higher uptake than nontumoral tissue. ${ }^{11} \mathrm{C}$-methionine (MET) is one of the most widely studied amino acid tracers for brain tumor imaging. The uptake of MET by recurrent tumors is associated with cellular proliferation and microvessel count and is different from uptake by radiation necrosis lesions, which is associated with passive diffusion across the broken BBB. Therefore, MET PET/CT has the potential to differentiate radiation injury from recurrent brain tumors. Previous studies have demonstrated that PWI might be similar or even superior to PET/CT for the differentiation of recurrence from radiation injury. ${ }^{8,9}$ However, each technique has its strengths and limitations, and validation in clinical trials is mandatory for the successful implementation of these techniques. $^{9-11}$

We conducted the present study to evaluate the usefulness of MET PET/CT and PWI for the differentiation of recurrence from radiation injury in patients with HGGs and quantitatively analyze the diagnostic values of MET PET/CT and PWI parameters. We also aimed to assess the combined diagnostic performance of the 2 modalities with regard to the therapeutic response of tumors.

\section{MATERIALS AND METHODS \\ Patient Population}

This retrospective study was approved by the ethics committee of Beijing Tiantan Hospital. The subjects were selected from a total of 235 patients with gliomas who underwent MET PET/CT at our institution between June 2015 and June 2017. The inclusion criteria were as follows: histopathologic diagnosis of HGGs (III-IV) according to the World Health Organization criteria; use of radiation therapy (3D conformal radiation therapy) or gamma knife surgery followed by tumor resection, with an interval of $>3$ months between radiation therapy and PET scanning; perfor- mance of contrast-enhanced and DSC-PWI, with an interval of $<1$ month between PET and PWI; and a clinical follow-up duration of $>3$ months. The exclusion criterion consisted of an unclear follow-up diagnosis. Ultimately, 42 patients were enrolled in our study.

\section{Determination of Lesions}

Clinical follow-up, follow-up MR imaging, or histopathologic analysis was performed to determine whether the lesions represented recurrence or radiation injury. Recurrence was defined by any of the following: progression of symptoms or death caused by brain tumor progression; a progressive increase (diameter increase, $>25 \%$ ) in the size of the contrast-enhancing lesion with or without antitumor treatment, which was consistent with an increase in the MET uptake area on follow-up MR imaging studies (with an interval of no less than 3 months), after the initial progress seen on the first follow-up MR imaging or new enhancing lesions on follow-up MR imaging; pathologic confirmation after tumor resection or biopsy; and progression of symptoms or a progressive increase in the lesion size (diameter increase, $<25 \%$ ) that could not be attributed to radiation injury or other disease. Radiation injury was diagnosed by pathologic examination or by a decrease in the size or stabilization of the contrast-enhancing lesion for a minimum of 6 months on follow-up MR imaging studies for patients without antitumor therapy.

\section{PET/CT Protocol and Analysis}

MET was synthesized by a solid-phase reaction. ${ }^{11} \mathrm{CO}_{2}$ was obtained by cyclotron (HM-10; Sumitomo, Hokkaido, Japan) and transferred to a carbon multifunctional synthesis module (CFN-C; Sumitomo, Tokyo, Japan). After methylation, a highly reactive methylation precursor, ${ }^{11} \mathrm{CH}_{3 \mathrm{I}}$, was obtained and then reacted with L-cysteine thioketone (Sigma, St. Louis, Missouri) to obtain MET at room temperature. MET was sterilized by passage through $0.22-\mu \mathrm{m}$ sterile filters before injection. PET/CT was performed using the Discovery Elite (GE Healthcare, Milwaukee, Wisconsin) 10 minutes after intravenous injection of 370-738.8 $\mathrm{MBq}$ of MET using the 3D acquisition mode for a 10-minute static scan. The data were reconstructed using the ordered subset expectation maximization algorithm, and the images were viewed on an AW4.5 Workstation (GE Healthcare).

Fused PET/CT images were visually assessed by 2 nuclear medicine physicians using contrast-enhanced brain MR images as references. Finally, the results of visual assessments were reclassified by consensus between 2 observers with respective experience in nuclear medicine of 6 and 13 years. The regional MET uptake was expressed as the standardized uptake value (SUV). An ROI for each lesion was outlined by $40 \%$ of the isocontour of the uptake maximum. In case no abnormality was detected on PET, the ROI was drawn on the area corresponding to the abnormality on MR imaging. As a normal control, an identical ROI was drawn over the contralateral cerebral cortex. The mean and maximum SUVs $\left(\mathrm{SUV}_{\text {mean }}\right.$ and $\mathrm{SUV}_{\text {max }}$, respectively) were measured for each ROI. The tumor-to-background $S_{\text {U }} V_{\text {max }}$ ratio $\left(T_{B R S U V}\right.$ max $)$ was defined as the ratio of the SUV $V_{\text {max }}$ of the lesion to the $S U V_{\text {max }}$ of the contralateral normal cortex. In case of multiple lesions, the lesion with the highest uptake was analyzed. 


\section{MR Image Acquisition and Analysis}

DSC-PWI was performed for all patients using an echo-planar imaging sequence during intravenous injection of the contrast agent. One of $2 \mathrm{MR}$ imaging devices was used (Tim Trio and Verio; Siemens, Erlangen, Germany). The PWI parameters were as follows: TR, 1400-1500 ms; TE, 30-32 ms; echo-train length, 1 $k$-space; position angle, $90^{\circ}$; matrix, $128 \times 128$. The MR imaging data were digitally transferred to a personal computer for further analyses. Dynamic curves were determined using a software package (Perfusion Mismatch Analyzer, Version 5.0.0.0, ASIST-Japan; http://asist.umin.jp/index-e.htm). CBV, TTP, MTT, and CBF maps were extracted using numeric integration of the curves. These maps, mainly CBF and CBV maps, were visually assessed by 2 physicians with respective experience in radiology of 7 and $>15$ years. ROIs of lesions were drawn on each slice of the CBV maps by connecting the dotted lines with the software using contrastenhanced MR images as a reference. Areas containing small vessels and necrosis were carefully excluded from the ROIs. Control ROIs were drawn in the contralateral normal region, excluding lesions or vessels. The relative parameters were calculated from the lesion-to-normal ratio. The mean $\mathrm{rCBV}$ ( $\left.\mathrm{rCBV}_{\text {mean }}\right)$ of lesions was calculated from the mean of the relative parameters for each slice. In case of multiple lesions, the lesion with the highest $\mathrm{rCBV}_{\text {mean }}$ was analyzed.

\section{Statistical Analysis}

Continuous parameters with normal distribution were presented as means \pm SDs. Mean differences were assessed between the 2 groups, and the significance of mean differences was evaluated using the Student $t$ test. The McNemar test was used to compare differences in the diagnostic performance between the 2 modalities. Logistic regression analysis was used to determine the best predictors of recurrence or radiation injury, and receiver operating characteristic analysis was performed to determine the best cutoff values for parameters that proved to be substantial predictors of recurrence or radiation injury. All statistical analyses were performed using SPSS software, Version 24 (IBM, Armonk, New York). We also determined the diagnostic performance of a combination of the best predictors. Areas under the receiver operating characteristic curves (AUCs) for different parameters were compared with the DeLong test ${ }^{12}$ using MedCalc for Windows (MedCalc Software, Mariakerke, Belgium). $P<.05$ indicated statistical significance.

\section{RESULTS}

The demographic and clinical characteristics of the included patients are shown in the On-line Table. Four of 42 patients were treated with temozolomide (patient Nos. 1, 11, 16, and 27). Of the 42 patients, 33 and 9 were diagnosed with recurrence and radiation injury, respectively, by histopathologic analysis ( $n=9$ and $n=1$, respectively) or clinical follow-up ( $n=24$ and $n=8$, respectively).

\section{Visual Assessments of MET PET/CT images}

Findings for 34 of the 42 patients were suggestive of recurrence, 6 scans were equivocal, and 2 scans were negative for recurrent tumors. When equivocal scans were reclassified as having negative findings, MET PET/CT showed a patient-based sensitivity
Table 1: Results of visual assessments of MET PET/CT for patients with HGGs

\begin{tabular}{lcccc}
\hline & \multicolumn{3}{c}{ Visual Assessment } & \\
\cline { 2 - 4 } Final Diagnosis & Positive & Equivocal & Negative & Total \\
\hline Recurrence & 30 & 3 & 0 & 33 \\
Radiation injury & 4 & 3 & 2 & 9 \\
Total & 34 & 6 & 2 & 42 \\
\hline
\end{tabular}

Table 2: Results of visual assessments of PWI for patients with HGGs

\begin{tabular}{lcccc}
\hline & \multicolumn{3}{c}{ Visual Assessment } & \\
\cline { 2 - 4 } Final Diagnosis & Positive & Equivocal & Negative & Total \\
\hline Recurrence & 22 & 8 & 3 & 33 \\
Radiation injury & 2 & 1 & 6 & 9 \\
Total & 24 & 9 & 9 & 42 \\
\hline
\end{tabular}

Table 3: Results of semiquantitative analysis of MET PET/CT and PWI parameters for the differentiation of recurrence from radiation injury in patients with HGGs

\begin{tabular}{lccc}
\multicolumn{3}{c}{ radiation injury in patients with HGGs } & \\
\cline { 2 - 3 } & \multicolumn{2}{c}{ Final Diagnosis } & \\
\hline SUV $_{\text {max }}$ & $5.10 \pm 2.41$ & $2.41 \pm 1.67$ & .003 \\
SUV $_{\text {mean }}$ & $2.83 \pm 1.27$ & $1.39 \pm 0.9$ & .003 \\
TBRSUV $_{\text {max }}$ & $3.48 \pm 1.17$ & $1.96 \pm 0.96$ & .003 \\
rCBV $_{\text {mean }}$ & $2.68 \pm 1.14$ & $1.33 \pm 0.77$ & .004 \\
\hline
\end{tabular}

${ }^{a}$ All $P$ values for discrimination between recurrence and radiation injury are significant.

and specificity of 0.909 and 0.556 , respectively, with corresponding positive and negative predictive values of 0.882 and 0.625 , respectively. Table 1 shows the findings of visual assessments of PET/CT images.

\section{Visual Assessments of DSC-PWI}

The findings of visual assessments of PWI are summarized in Table 2. Findings for 24 of the 42 patients were suggestive of recurrence, 9 scans were equivocal, and 9 were negative for recurrent tumors. When equivocal scans were reclassified as having negative findings, PWI showed a patient-based sensitivity and specificity of 0.667 and 0.778 , respectively, with corresponding positive and negative predictive values of 0.917 and 0.389 , respectively.

\section{Results of Semiquantitative Analysis}

Detailed data pertaining to semiquantitative analysis of imaging parameters for the 2 groups are summarized in Table 3. SUV $V_{\text {max }}$, $\mathrm{SUV}_{\text {mean }}, \mathrm{TBRSUV}_{\text {max }}$, and $\mathrm{rCBV}_{\text {mean }}$ were significantly higher for patients with recurrence than for patients with radiation injury. All the above parameters showed a significant discriminative power in receiver operating characteristic analysis (Fig 1). The optimal cutoff values for TBRSUV $\mathrm{Tmax}_{\text {max }}$ and $\mathrm{rCBV}_{\text {mean }}$ were determined as 1.85 and 1.83 , respectively. When 1.85 was used as the

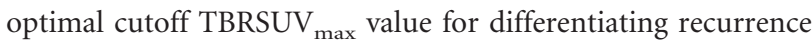
from radiation injury, the sensitivity and specificity of MET PET/CT were 0.970 and 0.667 , respectively. When 1.83 was used as the optimal cutoff value for $\mathrm{rCBV}_{\text {mean }}$, PWI showed a patientbased sensitivity and specificity of 0.788 and 0.889 , respectively. The McNemar test revealed statistically significant differences between TBRSUV $_{\text {max }}$ and $\mathrm{rCBV}_{\text {mean }}(P=.039)$. Disagreement between MET PET/CT and PWI was observed for 8 patients with recurrence and 4 with radiation injury (Figs 2 and 3). No patient 
with recurrence had negative findings on both MET PET/CT and perfusion-weighted images, while no patient with radiation injury showed positive findings on both image sets (Table 4).

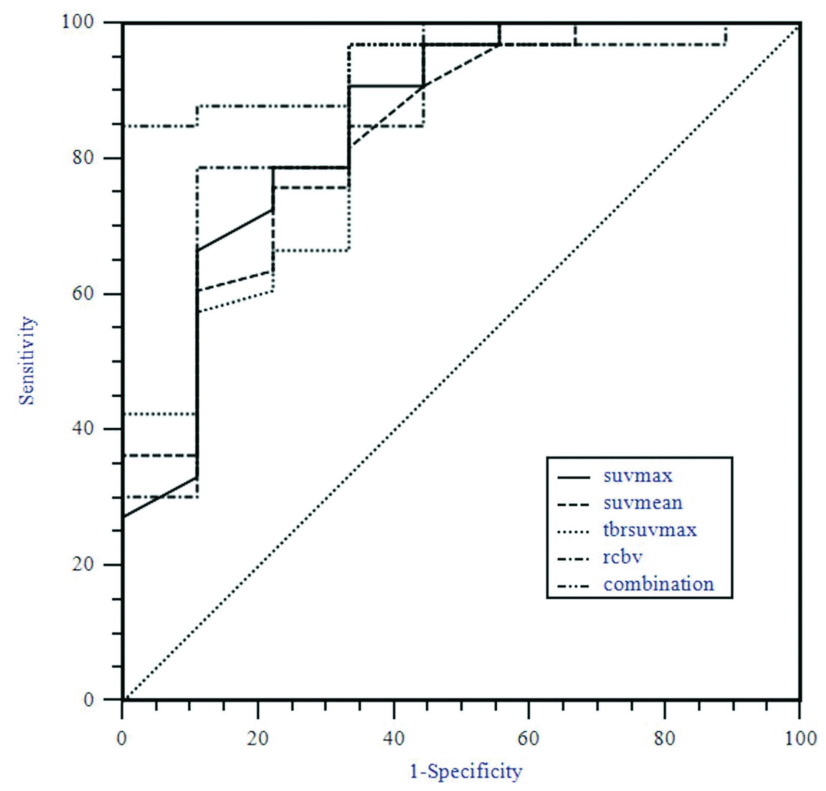

FIG 1. Receiver operating curves for perfusion-weighted MR imaging and MET PET/CT parameters for the differentiation of recurrence and radiation injury in patients with high-grade gliomas.

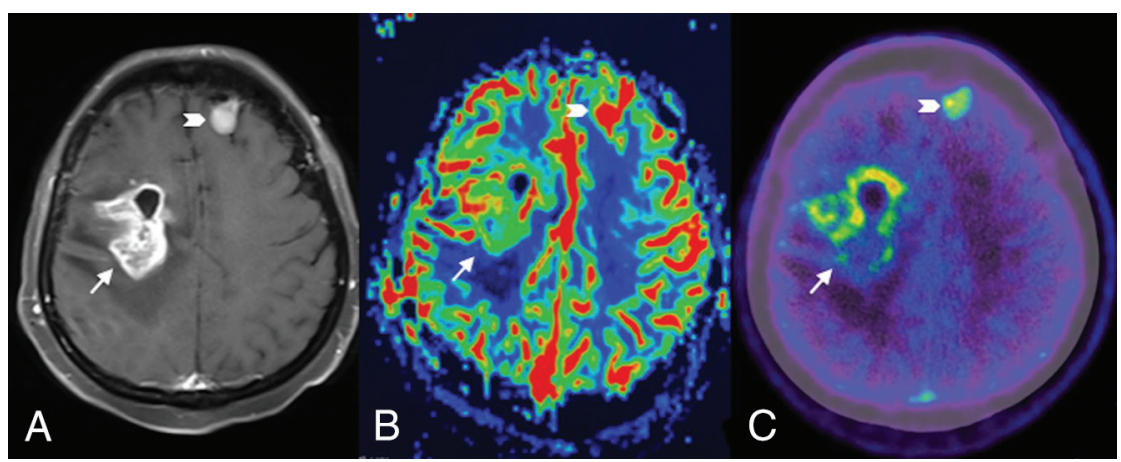

FIG 2. Contrast-enhanced T1-weighted MR image $(A)$ and a relative $C B V$ map $(B)$ and an MET PET/CT image (C) for a 41-year-old woman with recurrent glioblastoma. The lesion in the right frontal lobe (arrow) shows enhancement $(A)$ and positive findings on both the rCBV map $(B)$ and MET PET/CT image (C). The lesion localized in the left frontal lobe (chevron) might be a meningioma, which has been stable for several years.

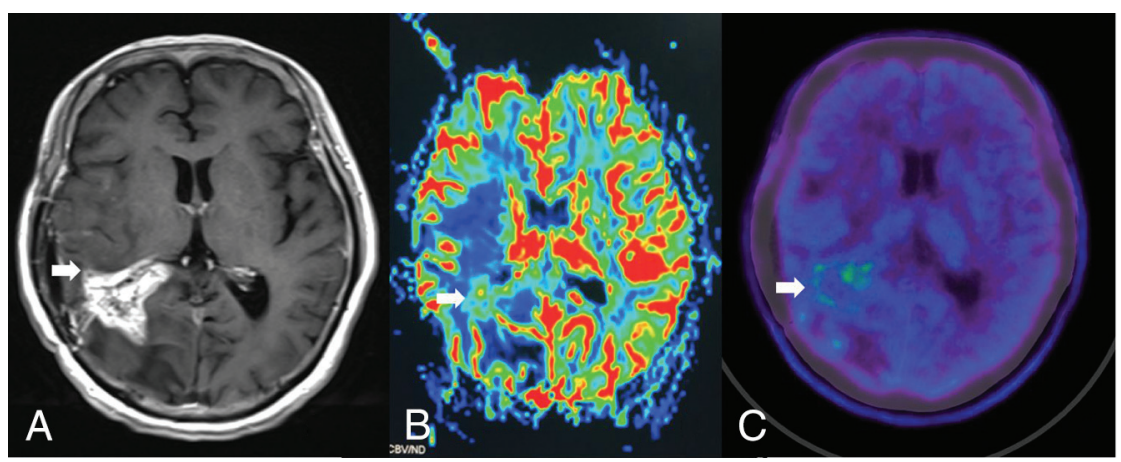

FIG 3. Contrast-enhanced T7-weighted MR image $(A)$ and relative $C B V$ map $(B)$ and MET PET/CT image $(C)$ for a 44-year-old man with necrosis. The lesion in the right parietal lobe (arrow) shows enhancement $(A)$ and negative findings on both the rCBV map (B) and the MET PET/CT image (C).

\section{DISCUSSION}

Agreement between visual assessments and semiquantitative analyses was observed for 37 of the $42(88 \%)$ patients when MET PET/CT was used and for 29 of the 42 (69\%) patients when PWI was used. The corresponding $\kappa$ coefficients were 0.595 and 0.355 , respectively.

AUCs for TBRSUV $\mathrm{Tmax}_{\text {max }}$ and $\mathrm{rCBV}_{\text {mean }}$ were $0.847 \pm 0.077$ and $0.845 \pm 0.078$, respectively, with no significant differences. Logistic regression and receiver operating characteristic analysis revealed the largest AUC $(0.953 \pm 0.031)$ for a combination of $\mathrm{TBRSUV}_{\text {max }}$ and $\mathrm{rCBV}_{\text {mean }}$, with corresponding sensitivity and specificity of 0.848 and 1.0 , respectively.

the present study, we evaluated the usefulness of MET /CT and PWI for the differentiation of recurrence from injury in patients with HGGs and quantitatively anthe diagnostic values of MET PET/CT and PWI parammilar findings were observed for the 2 modalities, and were combined.

Several studies have shown that MET PET/CT is useful for distinguishing tumor recurrence from radiation-induced necrosis. ${ }^{13-15}$ The major advantage of this technique is that the tracer is thought to accumulate preferentially in tumor tissue, resulting in good contrast against the normal tissue in the background. Similar to a previous study, ${ }^{16}$ all cases that showed negative results on visual assessment were those with radiation injury. Three cases of radiation injury showed equivocal findings and 4 showed positive findings on visual assessments; these findings can be explained by the mild MET uptake in some cases of necrosis. The mechanism underlying MET uptake by radiation necrosis lesions remains unclear. A possible mechanism is increased methionine metabolism and permeability induced by gliosis mediated by astrocytes and microglial cells, which are commonly observed in cases of radiation injury. ${ }^{16}$ Furthermore, radiation injury may break the $\mathrm{BBB}$, resulting in passive diffusion of MET, and this may explain the false-positive PET findings. Theoretically, the uptake by recurrence should exceed that by radiation injury lesions; however, one should not preclude the possibility of similar uptakes. According to suggestions in previous studies, ${ }^{17-19}$ we assumed that semiquantitative analysis of MET PET/CT parameters might be helpful in the present study, which showed that visual-assessment findings were not very different from those of semiquantitative analysis with a cutoff value for the differentiation of 


\begin{tabular}{|c|c|c|c|c|c|c|}
\hline \multirow[b]{3}{*}{ PET } & \multirow{2}{*}{\multicolumn{3}{|c|}{ Recurrence PWI }} & \multirow{2}{*}{\multicolumn{3}{|c|}{ Radiation Injury PWI }} \\
\hline & & & & & & \\
\hline & + & - & Total & + & - & Total \\
\hline+ & 25 & 7 & 32 & 0 & 3 & 3 \\
\hline - & 1 & 0 & 1 & 1 & 5 & 6 \\
\hline Total & 26 & 7 & 33 & 1 & 8 & 9 \\
\hline
\end{tabular}

Note:- + indicates TBRSUV $V_{\max } \geq 1.85$ or rCBV mean $\geq 1.83$; -, TBRSUV $\max <1.85$ or $\mathrm{rCBV}_{\text {mean }}<1.83$.

recurrence from radiation injury. According to our results, we expect that visual assessment may be useful for a rapid diagnosis.

It is difficult to determine the appropriate parameter to evaluate accumulation rates in the semiquantitative analysis of MET PET/CT. SUV produced a high SD, even in normal gray matter ${ }^{16}$; notably, TBR can reduce individual differences. Tsuyuguchi et $\mathrm{al}^{16}$ showed no significant difference between recurrence and necrosis groups with respect to TBR or SUV. Terakawa et $\mathrm{al}^{18}$ reported significant differences in all indices $\left(\mathrm{SUV}_{\text {mean }}, \mathrm{SUV}_{\text {max }}\right.$, and $\left.\mathrm{TBR}_{\text {mean }}\right)$, with the exception of the $\mathrm{TBR}_{\text {max }}$ between glioma recurrence and radiation necrosis. The difference may depend on the type and number of patients included in the study. The former involved a small number of patients, while the latter involved patients with both high- and low-grade gliomas. It has been shown that MET uptake in glioblastoma is higher than in low-grade gliomas, ${ }^{20}$ resulting in a better diagnostic ability for HGG recurrence than for recurrence of low-grade gliomas. Although the TBR has been used for differentiation, the cutoff ratio differs among studies, probably due to the variation of the PET scan protocol, which has not been standardized.

Terakawa et $\mathrm{al}^{18}$ reported that a mean lesion-to-normal uptake ratio of $>1.58$ resulted in the best sensitivity and specificity (75\% and $75 \%$, respectively) for the differentiation of recurrent gliomas (including HGGs and low-grade gliomas) from radiation necrosis. The reference region in the study of Terakawa et al was placed in the region of uptake in the contralateral normal frontal lobe gray matter, which was different from that in our study. In the present study, we used the ratio of the maximum lesion uptake to a reference uptake value instead of the mean uptake value. Previous studies using different cutoff values for the TBR to differentiate patients with glioma (all grades) recurrence from those with radiation injury have reported sensitivities ranging from $75 \%$ to $100 \%$ and specificities ranging from $60 \%$ to $100 \% .^{21,22}$

PWI can demonstrate the characteristics of the vascular physiology and the hemodynamics of tumors. Tumor recurrence is accompanied by abundant abnormal blood vessels with increased permeability, while necrosis is associated with treatment-induced vascular endothelial damage and coagulation necrosis. It has been reported that PWI correlated with vascularity could be used to differentiate recurrence from necrosis. In 2017, Patel et al ${ }^{5}$ summarized 28 articles on PWI-based differentiation of recurrent tumors and posttreatment changes and reported that the accuracy was similar among the best-performing DSC and dynamic contrast-enhanced parameters from each study. DSC and dynamic contrast-enhanced techniques each have their own limitations.
DSC has poorer spatial resolution and is more sensitive to the effects of susceptibility. Dynamic contrast-enhanced imaging requires complex pharmacokinetic models to maintain robustness with respect to nonlinearity between signal intensity and contrast agent concentration. The pooled sensitivities and specificities of the best-performing DSC-PWI parameters from all studies were $90 \%$ and $88 \%$. Within individual studies, PWI parameters distinguished viable tumor tissue from treatment changes with relatively good sensitivity and specificity. The most commonly evaluated PWI parameters are $\mathrm{rCBV}_{\text {mean }}$ and $\mathrm{rCBV}_{\text {max }}$. Patel et $\mathrm{al}^{5}$ also calculated the pooled sensitivities and specificities of $\mathrm{rCBV}_{\text {mean }}$ (threshold range, 0.9-2.15) and $\mathrm{rCBV}_{\text {max }}$ (threshold range, 1.49-3.1) for the detection of recurrence.

In the present study, we used a cutoff value of 1.83 for $\mathrm{rCBV}_{\text {mean }}$, which resulted in a sensitivity and specificity of 0.788 and 0.889 , respectively. Differences in cutoff values between our study and the previous studies could be attributed to differences in image-acquisition techniques, machines, processing techniques, and analysis tools. Young et $\mathrm{al}^{23}$ and Prager et $\mathrm{al}^{24}$ reported that $\mathrm{rCBV}$ individually showed the highest sensitivity and specificity to discriminate recurrence from radiation injury in patients with HGG when the cutoff values were 1.8 and 1.4, respectively. The present study showed that the agreement between visual assessments and semiquantitative analyses of parameters was better with MET PET/CT than with PWI in the discrimination of recurrence from radiation injury, probably because of the high background intensity in PWI that impaired accurate visual assessment.

Deng et $\mathrm{al}^{8}$ conducted a meta-analysis of 11 studies assessing MET PET/CT and 7 studies assessing DSC-PWI and showed that the AUC for PWI was larger than that for MET PET/CT $(P<.01)$. Another meta-analysis ${ }^{9}$ conducted in 2010 also reported that PWI may be superior to MET PET/CT for the differentiation of recurrence from radiation necrosis. Various types and grades of gliomas included in the meta-analysis may cause bias. In contrast, the present study derived similar AUCs for PWI and MET PET/CT. These discrepant findings could be attributed to differences in acquisition parameters and PWI being performed using 2 different MR imaging units in the present study. Although receiver operating characteristic curve analysis with an rCBV of $>3.69$ representing tumor recurrence showed $100 \%$ sensitivity and $100 \%$ specificity, Kim et $\mathrm{al}^{9}$ failed to show statistical significance in the HGG diagnostic accuracy of PWI and MET due to the small number of analyzed cases $(n=10)$. D'Souza et $\mathrm{al}^{25}$ compared MET PET/CT and advanced MR imaging (inclusion of MR spectroscopy and PWI) in the identification of tumor recurrence in 41 patients with HGGs; that study indicated that MET PET seemed more sensitive, whereas advanced MR imaging seemed more specific. There was no statistically significant difference in the diagnostic performance of either technique. Similar to the present study, the diagnosis of recurrence was determined by histology or follow-up. Concordant results of PWI, MET PET, and histology were shown in 7 cases with HGGs in the study by Dandois et $\mathrm{al}^{26}$; not all patients had a histologic diagnosis. This study was analyzed on the basis of the imaging process, and 31 of 33 combined MR and PET studies showed same diagnostic results.

Our study was based on patients, and 12/42 cases of disagree-

AJNR Am J Neuroradiol 40:253-59 Feb 2019 www.ajnr.org 
ment were observed between MET PET and PWI. Most interesting, all cases showing negative findings in both imaging modalities were those of radiation injury, while all cases showing positive findings in both imaging modalities were those of recurrence. Collectively, these findings suggest that agreement of MET PET/CT and PWI may be helpful in the determination of recurrence. In case of disagreement between MET PET/CT and PWI findings, the diagnosis may be unclear. In addition, MET PET/CT in this study has a higher sensitivity than PWI but exhibits a lower specificity; this may be related to the cutoff value selected. We found that the AUC for a combination of TBRSUV $V_{\max }$ and $\mathrm{rCBV}_{\text {mean }}$ was the largest, though there was no significant difference. This finding suggests that the accuracy of a combination of MET PET/CT and PWI parameters is better than that of individual parameters for the differentiation of radiation necrosis and recurrence in patients with HGGs.

Our effort to determine which of the 2 methods is better was inconclusive; good use of each method is effective. PET/MR imaging combines 2 imaging modalities and has the advantages of high spatial resolution and numerous biologically relevant tracers. However, the method for PET/MR imaging attenuation correction, which affects quantitative reliability, has yet to be optimized. Rausch et $\mathrm{al}^{27}$ proposed that ultrashort TE-based and BD-based attenuation correction (BD, noncommercial prototype software by Siemens Healthcare) is clinically acceptable for SUV calculations of tracer uptake in lesions within the brain. In addition, the more recent developments in attenuation correction for MR imaging-based methods (eg, RESOLUTE) are promising. ${ }^{28}$ However, the use of PET/MR imaging has some unclear drawbacks: Patient comfort is decreased and communication with the patient is hampered. Thus, movement artifacts are more common in PET/MR imaging. Although the application of PET/MR imaging may play a greater role in the combination of PET and advanced MR imaging using hybrid systems, the drawbacks of PET/MR imaging still need to be overcome.

The findings of this study suggest that MET PET/CT and PWI are equally accurate in the differentiation of recurrence from radiation injury in patients with HGGs, and a combination of the 2 modalities could be helpful.

This study has some limitations. First, it was a retrospective study. Second, histopathologic analysis was not performed for all patients. Third, PWI was performed using 2 different machines with varied acquisition parameters. Fourth, the proportion of patients with radiation injury in our study population was small. Thus, our results should be interpreted with caution.

\section{CONCLUSIONS}

This study compared MET PET/CT and PWI for the differentiation of recurrence from radiation injury in patients with HGGs. Both imaging techniques had similar diagnostic performance in discriminating recurrence and radiation injury, and combining the 2 may be helpful. Meanwhile, semiquantitative analysis can be helpful in cases in which visual assessment yields equivocal results. Further studies with a larger sample size, a better study design, and using machines with identical image-acquisition parameters are warranted to confirm our results.

\section{REFERENCES}

1. Verma N, Cowperthwaite MC, Burnett MG, et al. Differentiating tumor recurrence from treatment necrosis: a review of neuro-oncologic imaging strategies. Neuro Oncol 2013;15:515-34 CrossRef Medline

2. Hyare H, Thust S, Rees J. Advanced MRI techniques in the monitoring of treatment of gliomas. Curr Treat Options Neurol 2017;19:11 CrossRef Medline

3. Galldiks N, Law I, Pope WB, et al. The use of amino acid PET and conventional MRI for monitoring of brain tumor therapy. Neuroimage Clin 2017;13:386-94 CrossRef Medline

4. Mangla R, Singh G, Ziegelitz D, et al. Changes in relative cerebral blood volume 1 month after radiation-temozolomide therapy can help predict overall survival in patients with glioblastoma. Radiology 2010;256:575-84 CrossRef Medline

5. Patel P, Baradaran H, Delgado D, et al. MR perfusion-weighted imaging in the evaluation of high-grade gliomas after treatment: a systematic review and meta-analysis. Neuro Oncol 2017;19:118-27 CrossRef Medline

6. Barajas RF Jr, Chang JS, Segal MR, et al. Differentiation of recurrent glioblastoma multiforme from radiation necrosis after external beam radiation therapy with dynamic susceptibility-weighted contrast-enhanced perfusion MR imaging. Radiology 2009;253:486-96 CrossRef Medline

7. Bisdas S, Naegele T, Ritz R, et al. Distinguishing recurrent highgrade gliomas from radiation injury: a pilot study using dynamic contrast-enhanced MR imaging. Acad Radiol 2011;18:575-83 CrossRef Medline

8. Deng SM, Zhang B, Wu YW, et al. Detection of glioma recurrence by ${ }^{11} \mathrm{C}$-methionine positron emission tomography and dynamic susceptibility contrast-enhanced magnetic resonance imaging: a metaanalysis. Nucl Med Commun 2013;34:758-66 CrossRef Medline

9. Kim YH, Oh SW, Lim YJ, et al. Differentiating radiation necrosis from tumor recurrence in high-grade gliomas: assessing the efficacy of 18F-FDG PET, 11C-methionine PET and perfusion MRI. Clin Neurol Neurosurg 2010;112:758-65 CrossRef Medline

10. Hojjati M, Garg V, Badve CA, et al. Differentiation of recurrent spinal ependymoma from postradiation treatment necrosis through multiparametric PET-MR and perfusion MRI. Clin Imaging 2017;41:48-52 CrossRef Medline

11. Wang $\mathrm{X}, \mathrm{Hu} \mathrm{X}, \mathrm{Xie} \mathrm{P}$, et al. Comparison of magnetic resonance spectroscopy and positron emission tomography in detection of tumor recurrence in posttreatment of glioma: a diagnostic metaanalysis. Asia Pac J Clin Oncol 2015;11:97-105 CrossRef Medline

12. DeLong ER, DeLong DM, Clarke-Pearson DL. Comparing the areas under two or more correlated receiver operating characteristic curves: a nonparametric approach. Biometrics 1988;44:837-45 CrossRef Medline

13. Nihashi T, Dahabreh IJ, Terasawa T. Diagnostic accuracy of PET for recurrent glioma diagnosis: a meta-analysis. AJNR Am J Neuroradiol 2013;34:944-50, S1-11 CrossRef Medline

14. Minamimoto R, Saginoya T, Kondo C, et al. Differentiation of brain tumor recurrence from post-radiotherapy necrosis with $11 \mathrm{C}$-methionine PET: visual assessment versus quantitative assessment. PLoS One 2015;10:e0132515 CrossRef Medline

15. Skvortsova TY, Brodskaya ZL, Gurchin AF. PET using 11C-methionine in recognition of pseudoprogression in cerebral glioma after combined treatment [in English, Russian]. Zh Vopr Neirokhir Im NN Burdenko 2014;78:50-58 CrossRef Medline

16. Tsuyuguchi N, Takami T, Sunada I, et al. Methionine positron emission tomography for differentiation of recurrent brain tumor and radiation necrosis after stereotactic radiosurgery: in malignant glioma. Ann Nucl Med 2004;18:291-96 CrossRef Medline

17. Herrmann K, Czernin J, Cloughesy T, et al. Comparison of visual and semiquantitative analysis of 18F-FDOPA-PET/CT for recurrence detection in glioblastoma patients. Neuro Oncol 2014;16:603-09 CrossRef Medline

18. Terakawa Y, Tsuyuguchi N, Iwai Y, et al. Diagnostic accuracy of 
11C-methionine PET for differentiation of recurrent brain tumors from radiation necrosis after radiotherapy. J Nucl Med 2008;49: 694-99 CrossRef Medline

19. Martínez-Amador N, Jimenez-Bonilla J, Martínez-Rodriguez I, et al. Value of the visual and semiquantitative analysis of carbon-11-methionine PET/CT in brain tumors' recurrence versus post-therapeutic changes. Nucl Med Commun 2017;38:1125-32 CrossRef Medline

20. Kaschten B, Stevenaert A, Sadzot B, et al. Preoperative evaluation of 54 gliomas by PET with fluorine-18-fluorodeoxyglucose and/or carbon-11-methionine. J Nucl Med 1998;39:778-85 Medline

21. Tripathi M, Sharma R, Varshney R, et al. Comparison of F-18 FDG and C-11 methionine PET/CT for the evaluation of recurrent primary brain tumors. Clin Nucl Med 2012;37:158-63 CrossRef Medline

22. Deuschl C, Kirchner J, Poeppel TD, et al. ${ }^{11} \mathrm{C}-\mathrm{MET}$ PET/MRI for detection of recurrent glioma. Eur J Nucl Med Mol Imaging 2018;45: 593-601 CrossRef Medline

23. Young RJ, Gupta A, Shah AD, et al. MRI perfusion in determining pseudoprogression in patients with glioblastoma. Clin Imaging 2013;37:41-49 CrossRef Medline
24. Prager AJ, Martinez N, Beal K, et al. Diffusion and perfusion MRI to differentiate treatment-related changes including pseudoprogression from recurrent tumors in high-grade gliomas with histopathologic evidence. AJNR Am J Neuroradiol 2015;36:877-85 CrossRef Medline

25. D'Souza MM, Sharma R, Jaimini A, et al. ${ }^{11} \mathrm{C}-\mathrm{MET}$ PET/CT and advanced MRI in the evaluation of tumor recurrence in high-grade gliomas. Clin Nucl Med 2014;39:791-98 CrossRef Medline

26. Dandois V, Rommel D, Renard L, et al. Substitution of 11C-methionine PET by perfusion MRI during the follow-up of treated highgrade gliomas: preliminary results in clinical practice. J Neuroradiol 2010;37:89-97 CrossRef Medline

27. Rausch I, Rischka L, Ladefoged CN, et al. PET/MRI for oncologic brain imaging: a comparison of standard MR-based attenuation corrections with a model-based approach for the Siemens mMR PET/MR System. J Nucl Med 2017;58:1519-25 CrossRef Medline

28. Ladefoged CN, Andersen FL, Kjaer A, et al. RESOLUTE PET/MRI attenuation correction for $\mathrm{O}-\left(2-{ }^{18} \mathrm{~F}\right.$-fluoroethyl)-L-tyrosine (FET) in brain tumor patients with metal implants. Front Neurosci 2017;11:453 CrossRef Medline 